\title{
Les idées éducatives de Spencer en France
}

\section{Dominique Ottavi}

\section{(2) OpenEdition}

\section{Journals}

Édition électronique

URL : http://journals.openedition.org/aes/284

DOI : $10.4000 /$ aes.284

ISSN : 2258-093X

\section{Éditeur}

Laboratoire LISAA

\section{Référence électronique}

Dominique Ottavi, «Les idées éducatives de Spencer en France », Arts et Savoirs [En ligne], 4 | 2014 mis en ligne le 15 mai 2014, consulté le 19 avril 2019. URL : http://journals.openedition.org/aes/284 ; DOI : 10.4000/aes.284

Ce document a été généré automatiquement le 19 avril 2019

Centre de recherche LISAA (Littératures SAvoirs et Arts) 


\section{Les idées éducatives de Spencer en France}

\section{Dominique Ottavi}

Herbert Spencer a exercé une importante influence en France dans le domaine de l'éducation. Cet aspect assez marginal de sa vaste philosophie synthétique reflète cependant un intérêt intense et constant pour les questions éducatives et pour le rôle de l'école dans la société. En effet, si Spencer est revenu souvent sur cette question, l'œuvre qui traite ce sujet de manière centrale est De l'éducation intellectuelle, morale et physique ${ }^{1}$, ouvrage paru initialement en 1861 et traduit en français en 1879; il réunit des essais parus entre 1849 et 1859 . Cette œuvre est précoce, et peut être considérée comme mineure dans la carrière philosophique de Spencer, cela ne l'empêche pas d'avoir marqué, en France, la pensée républicaine de la Troisième République et d'être l'un des premiers livres qui illustrent le propos de Félix Pécaut : «La France pédagogise $»^{2}$. En effet, la mise en place de l'instruction obligatoire et de l'école publique dans les années 1880 s'accompagne d'un vaste mouvement de réflexion pédagogique. Si tout enfant doit bénéficier de l'école élémentaire, réfléchir au «comment» de cette généralisation devient une urgence, au point que Pécaut dénonce là une sorte de mode, d'excès, de confiance excessive dans la rationalisation des pratiques. Comme si la pédagogie ou la question des moyens de l'éducation et de la transmission pouvait faire l'objet d'une science, promettre la réussite du vaste projet d'instruction publique et d'éducation nationale. Cette opposition ancienne des tenants d'un «art " de l'éducation, et de ceux qui pensent que la pédagogie peut connaître un progrès nourri d'avancées scientifiques, se poursuit jusqu'à nos jours mais est souvent, hélas, négligée par les épistémologues. Spencer, dans la terminologie en usage actuellement dans ces débats, serait donc à ranger parmi les "pédagogues ", parmi ceux qui ont cru au progrès de l'éducation dans le cadre d'un progrès général des sciences. La traduction de De l'éducation, il faut le souligner, sera largement diffusée dans les Écoles Normales d'Instituteurs pendant la Troisième République, et constituera un élément de la réflexion sur le renouvellement des méthodes et sur le progrès en pédagogie, que l'on croit, souvent et à tort, absente des formations de cette époque. 
2 Quel était le rapport de Spencer à l'éducation? Il faut d'abord rappeler son cas personnel et son éducation particulière, du fait qu'il était le seul survivant de sa fratrie. Pourquoi survivre parmi tant de victimes de la mortalité infantile? Spencer a eu sans doute le sentiment d'une force impersonnelle habitant les individus, d'une puissance de vie antérieure à la conscience, de ce qu'il nommera dans ses Premiers principes ${ }^{3}$ la Force ${ }^{4}$. Un autre facteur d'ordre personnel de son intérêt pour l'éducation est que son père était instituteur et adepte des théories de Pestalozzi ${ }^{5}$. Il a donc été tôt sensibilisé aux questions éducatives et informé des théories modernes en la matière. En plein XIx ${ }^{e}$ siècle, Rousseau et ses continuateurs représentent encore la pointe acérée de la théorie éducative. Spencer de ce point de vue est héritier de cette modernité de la fin du XVIII ${ }^{\mathrm{e}}$ siècle qui s'éloigne, et il la relie à la pensée scientifique du XIX ${ }^{e}$ siècle, notamment à la théorie évolutionniste.

3 On retrouve dès cette première réflexion sur l'éducation des thèmes qui seront développés plus tard dans toute la philosophie de Spencer: les organismes individuels, humanité comprise, sont soumis à la concurrence, à la lutte pour la vie, en vue de la sélection des plus aptes, «the survival of the fittest». Cette vision conduit à donner beaucoup d'importance, comme dans la philosophie de Lamarck, à l'idée d'effort. Il ne suffit pas pour un organisme de posséder des qualités innées, il faut encore les mettre en valeur et pour cela, lutter, persévérer. Il est important de distinguer cette idée centrale du concept de «sélection naturelle» de Darwin. C'est Spencer qui a influencé Darwin, et ce dernier a ajouté l'idée de la "survivance du plus apte», empruntée à Spencer, à la cinquième édition de l'Origine des espèces en 1869. Mais la sélection naturelle est un concept différent de cette concurrence chère à Spencer, ne serait-ce que parce qu'elle fait intervenir la capacité reproductive qui ne tient pas uniquement à la "supériorité » intrinsèque d'un organisme.

\section{Quelle modernité pour De l'éducation?}

4 La composition du recueil De l'éducation permet déjà de situer les engagements de Spencer, et la modernité de son point de vue, si l'on entend par là le rejet des traditions et des habitudes.

5 L'ensemble, on l'a dit, est composé d'essais, préalablement publiés dans des revues entre 1849 et 1859. La première partie, intitulée "Quel est le savoir le plus utile? ", milite pour l'enseignement des sciences et pour un renouvellement des programmes d'études, principalement au nom du fait que les applications des sciences sont utiles à la vie, et aussi parce que les sciences sont la conquête la plus aboutie du progrès. Alors que la question de savoir si Spencer appartient vraiment au positivisme est toujours discutée, cette conviction l'y rattache. On peut signaler ici que l'ensemble des discussions soulevées par la place respective des sciences et des humanités dans l'enseignement secondaire trouvera une concrétisation en France dans la réforme de 1902 qui institue l'enseignement «moderne» dans les lycées. Le second essai, "De l'éducation intellectuelle ", expose les meilleures méthodes d'apprentissage en préconisant le recours à l'activité de l'élève et aux expériences concrètes, méthodes « actives » avant la lettre. Il s'agit là d'une interprétation des idées du grand pédagogue suisse Pestalozzi. Le troisième essai, intitulé "De l'éducation morale » propose une conception très originale de la discipline « libérale » et de la formation du caractère, inspirée, cette fois, de Jean-Jacques Rousseau lui-même. Quant au quatrième essai, « De l'éducation physique », il plaide pour 
une éducation qui donne davantage de place au corps, se soucie davantage de la santé et de la force physique. Ce dernier essai qui compare les enfants aux animaux d'élevage, préconise pour leur santé de les laisser manger ce qu'ils veulent, de se vêtir selon leurs besoins, de les laisser jouer autant qu'ils le désirent. Ces préconisations valent également pour les filles. Ce dernier essai, dans la simplicité de ses recommandations et de leur justification, livre quelques idées essentielles: la philosophie de l'éducation est ici soutenue par l'idée générale d'intégration de l'homme à la nature. Vivant parmi les autres, l'homme doit, dans sa jeunesse, faire la preuve de son aptitude à la survie et de ses qualités pour la lutte. C'est dans le dessein de laisser opérer la nature que le «laisserfaire ", pour reprendre l'expression des économistes libéraux, est présenté comme la règle par excellence de la méthode d'éducation.

La question essentielle à laquelle l'ouvrage se donne pour but de répondre peut être formulée ainsi : quelle éducation convient à l'époque nouvelle que nous vivons? Cette question ne pourrait-elle se trouver dans n'importe lequel de nos quotidiens ou hebdomadaires actuels ? Il n'est pas anachronique d'opérer ce rapprochement, parce que Spencer est l'inventeur d'une nouvelle manière de voir. Il a en tout cas contribué à ce que des questions éthiques et politiques ne soient plus traitées en référence à des valeurs absolues, mais à une évolution réputée inéluctable à laquelle le présent est sommé de s'adapter.

7 En vertu de la tendance à la complexification de l'évolution en général, la vie sociale devient elle aussi de plus en plus complexe; il faut noter à ce propos que Spencer, bien qu'il ait été vivement critiqué par Durkheim, a préparé la théorisation par ce dernier de la division sociale du travail. En effet, c'est l'application du principe de la marche de l'évolution du simple vers le complexe qui a inspiré à Durkheim sa réflexion sur la complexification du travail dans la société industrielle. Selon Spencer, cette société complexe constitue pour l'individu un milieu auquel il doit s'adapter, dans lequel il doit trouver sa place. Dans ces conditions, par définition toujours nouvelles au fur et à mesure que les générations se succèdent, une éducation qui conforme l'individu à un modèle, qui le fait ployer sous le poids de l'autorité, ne peut être que contraire à l'intérêt général et, à long terme, à l'intérêt de l'espèce humaine. Elle risque de fausser les conditions du développement de l'individu, l'empêcher de faire valoir ses qualités propres dans la concurrence vitale et sociale qui est le moteur du progrès. C'est dans le sillage de cette idée que Spencer se réapproprie l'héritage des novateurs que sont Jean-Jacques Rousseau et son disciple Pestalozzi, et qu'il développe une vision particulière de la discipline, la théorie des « réactions naturelles »

8 En ce qui concerne le premier point, on remarque à la lecture de l'ouvrage De l'éducation que de nombreux passages constituent une reprise des idées de Rousseau et de Pestalozzi ; Spencer vante l'« éducation négative », et approuve aussi l'enseignement par les «choses » ou l'« enseignement par l'aspect » concrétisé par Pestalozzi ${ }^{7}$. Il considère qu'il faut donner libre cours au désir de mouvement de l'enfant, à sa parole, à sa curiosité, en forgeant en même temps une conception nouvelle du rôle de l'enseignant. Or, si les propos de Spencer peuvent être calqués sur ceux de Rousseau, il n'en demeure pas moins que l'interprétation qu'il en fait est forcée, car le concept de nature auquel ils réfèrent est profondément différent.

9 Pour Rousseau, l'homme naturel que l'on retrouve dans l'enfant est un homme épargné par l'influence de la civilisation, c'est un être en possession de toutes ses facultés, dont la raison est intacte ainsi que les sentiments moraux. Pour Spencer, la « nature » humaine 
n'est pas un état originel préservé au fond de l'individu, c'est un mouvement permanent, une évolution. L'homme naturel se modifie donc, et la société fait partie des nouvelles circonstances dans lesquelles il doit vivre, et la notion de "dépravation" chère à Rousseau, qui signifie que l'homme est détourné de sa nature par la société, ne saurait avoir de sens pour Spencer. L'environnement géographique ou physique et l'environnement social sont, de son point de vue, aussi naturels l'un que l'autre. L'influence de la société ne saurait donc entrer en conflit avec la nature. En revanche, vouloir lutter contre l'évolution de cette société et de l'espèce humaine est pour lui un projet dangereux et utopique. Loin de vouloir changer la société pour orienter les relations sociales vers plus de justice et d'égalité, il privilégie la préparation de l'individu à une situation de concurrence et d'inégalité :

La lutte pour l'existence est si vive dans les temps modernes, que peu nombreux seront les hommes qui pourront en sortir vainqueurs. Déjà des milliers d'entre eux succombent sous la trop haute pression qu'ils subissent. [...] Il devient donc d'une importance particulière d'élever les enfants de façon, non-seulement qu'ils soient aptes à soutenir la lutte intellectuelle qui les attend, mais aussi qu'ils puissent supporter physiquement l'excessive fatigue à laquelle ils seront soumis. ${ }^{8}$

On est bien loin de l'idéal rousseauiste, dans lequel l'Émile, traité d'éducation, complète le Contrat social, projet politique, les deux poursuivant le but de refonder l'ordre social. Dans l'évolutionnisme spencérien, trop d'intervention de l'État, trop d'éducation, trop d'autorité, etc., empêchent les meilleurs de manifester leur supériorité et les plus faibles de payer le prix de leur infériorité. Spencer nous invite ainsi à considérer les conséquences de la liberté dans la société telle qu'elle est, devant lui, en pleine révolution industrielle, et non pas dans la société telle qu'elle devrait être. On a là les conséquences d'un rousseauisme qui a renoncé à changer la société, pour limiter ses ambitions à une action sur l'individu et l'enfant, et qui va prôner une pédagogie libertaire dont la finalité est, à travers l'épanouissement individuel, la poursuite du progrès en général.

\section{L'école, le maître, la morale}

11 Dans ce contexte, quel peut être le rôle de l'éducation, de l'école, et en particulier de cette méthode disciplinaire que Spencer nomme système des "réactions naturelles ${ }^{9}$ ? L'éducation en général doit ménager la nature de l'enfant, et, contre une pédagogie traditionnelle contraignante, uniformisante, niveleuse, elle doit laisser s'exprimer la diversité des talents. Cependant, l'éducation au sein de la famille a toutes les chances, elle aussi, de modeler l'enfant selon son milieu social, de «reproduire» ce dernier, pour reprendre la terminologie de Pierre Bourdieu. Pour éviter ce processus que Spencer considère comme une entrave à l'expression des différences naturelles individuelles, l'école peut être un recours en constituant un lieu indépendant où l'enfant sera considéré pour lui-même, en tant qu'individu singulier, de manière à permettre en quelque sorte un traitement égalitaire des différences. Le système dit des réactions naturelles se justifie par ce principe. Il faut que les enfants soient laissés libres de réaliser un certain nombre d'expériences, pour qu'ils puissent évaluer les conséquences de leur comportement. Leur intelligence s'exerce ainsi dans une réflexion sur l'expérience, tandis que la moralité se forme de la seule manière que Spencer juge légitime, c'est-à-dire dans la confrontation avec le milieu naturel et humain. ${ }^{10}$ Contre l'inculcation des normes et des croyances, l'esprit de l'élève est renvoyé à lui-même et à l'échange avec ses pairs pour confronter la diversité des points de vue, et en tirer la leçon. Autrement dit, la liberté d'agir comporte 
aussi des risques, et c'est le point qui fera réagir le plus les lecteurs contemporains de Spencer. On peut souligner ici que ce type d'éducation consiste moins en un apprentissage résultant de l'expérience, selon la définition rousseauiste de l'éducation des "choses ", qu'en une confrontation des capacités héréditaires de l'individu, de son acquis biologique, avec les épreuves de l'environnement ; l'« éducation négative » pour Spencer équivaut au bon déroulement de ce processus. L'expérience est pour Spencer une sorte de "test » à grande échelle, qui sélectionne selon lui les meilleurs, qui permet le dégagement d'une élite selon des critères naturalistes.

Qu'en est-il alors de l'enseignant? Il est renvoyé à un rôle d'organisation et d'accompagnement plutôt qu'à un rôle d'éducation et d'enseignement. Ce point de vue résonne étrangement avec nos préoccupations contemporaines. En effet, des voix de plus en plus nombreuses s'élèvent aujourd'hui pour dénoncer la perte de l'autorité, pour déplorer l'impuissance des enseignants à imposer les acquisitions et les règles de comportement. Mais en même temps, le métier est tout à fait officiellement orienté vers l'accompagnement et l'encadrement de l'activité individuelle. Au fond, on peut se demander si cette tension dans l'institution scolaire contemporaine n'est pas le corrélat de la présence inconsciente d'idées spencériennes. Et si l'autorité de l'adulte est affaiblie, si son rôle d'éducateur est mal défini, ne devient-il pas le spectateur impuissant d'une sorte de concurrence vitale? Un certain discours des adultes qui consiste à invoquer, face à des situations conflictuelles et violentes, la construction de l'autonomie et la nécessité pour les jeunes de "gérer" la réalité qu'ils vivent, ne relève-t-il pas alors de ce biologisme informulé ? Dans ce cas, Spencer ne serait pas seulement un philosophe ultralibéral prêt à sacrifier la dignité humaine au nom du progrès, il serait aussi le devin qui force ÆEdipe à voir ce qu'il ne veut pas voir, c'est-à-dire l'application d'un certain naturalisme social au sein de l'institution scolaire, qui s'exprime souvent par cette formule : « il faut bien que les enfants s'adaptent, la vie n'est pas rose ».

La réflexion pourrait être poursuivie par une interrogation au sujet de la neutralité dans le domaine des valeurs. Spencer, dans la continuité de ses écrits sur l'éducation, a développé une critique rigoureuse de la morale, et nous pose ainsi le problème d'une laïcité qui tend à une complète neutralité en matière de principes pratiques, laissant ainsi libre cours à une morale qui résulte d'un simple bilan de l'expérience. Dans son Introduction à la science sociale en 1873, Spencer consacre un chapitre aux «préjugés de l'éducation $»^{11}$. Il y relève que l'éducation des enfants est pétrie de contradictions dans le domaine moral: d'un côté on leur inculque une morale chrétienne, une religion de l'amour et du sacrifice, et de l'autre, en particulier par le biais des textes antiques, on leur inculque une religion de la haine qui leur enjoint de sacrifier les autres. Par conséquent, Spencer suggère de supprimer ces sources contradictoires de la morale, de supprimer les humanités aussi bien que l'enseignement chrétien, pour les remplacer par la science, et par le juste équilibre entre amour et haine que révèle par exemple la théorie de l'évolution. Mieux vaut reconnaitre les ressorts réels de la conduite, au lieu de se bercer d'illusions fondées sur la transcendance. Spencer interprète toute tentative de penser une différence entre le droit et le fait, entre le principe et la réalité, entre l'idéal et sa réalisation, comme une hypocrisie, un refus de reconnaitre la morale naturelle. L'abstention totale dans le domaine moral, alliée à la proclamation de quelques grands principes abstraits, n'est-elle pas la toile de fond de la crise de la transmission des valeurs, et n'a-t-on pas tendance à renvoyer les jeunes à leur "esprit critique " ou à leur 
«discernement » lorsque se posent des problèmes d'évaluation morale ou de rapport à une norme ? Là encore, la position de Spencer prend valeur de signe.

\section{Les idées éducatives de Spencer et la pédagogie en France}

14 En France, on a surtout vu dans les écrits de Spencer un plaidoyer en faveur de la liberté individuelle. La fascination pour la science, l'espoir de voir l'éducation établie sur les bases fermes de la biologie expliquent aussi le succès de sa théorie éducative. Rappelons que la traduction par Théodule Ribot et Alfred Espinas en 1875 de ses Principes de psychologie, parus initialement en 1855 , en a fait un élément incontournable de la psychologie expérimentale ${ }^{12}$. Quant au traducteur de son ouvrage sur l'éducation, Alexis Bertrand, il diffuse et soutient la philosophie spencérienne, car il y voit un possible appui scientifique pour les idées libertaires. Mais les travaux de Spencer sont aussi diffusés par la Revue pédagogique, qui parait à partir de 1876. Ceci est significatif car c'est la revue d'éducation par excellence, le reflet des choix politiques et pédagogiques du ministère ${ }^{13}$, et une sorte de fidélité à Spencer s'y manifeste. Ainsi, en 1880, Paul Souquet ${ }^{14} \mathrm{y}$ résume honnêtement l'ouvrage sur l'éducation de Spencer, sans y ajouter de commentaire particulier, comme s'il s'agissait avant tout d'informer les lecteurs au sujet d'une œuvre incontournable. En 1911 encore, l'inspecteur Henri Gazin fait l'éloge du système des "réactions naturelles $»^{15}$. Le Dictionnaire de pédagogie de Ferdinand Buisson lui consacre un article dans sa deuxième édition, celle de 1911 (la première datant de 1887); l'article, situé dans cette somme de la pensée pédagogique destinée aux enseignants, est particulièrement révélateur de cette consécration. L'auteur en est le médecin Georges Poyer.

15 On peut ici glisser une remarque supplémentaire : ceux qui prétendent encore que la Troisième République représente en matière d'éducation l'installation de normes pesantes et d'une tradition répressive ${ }^{16}$ devraient prendre en considération ce type de texte où l'on perçoit un grand souci d'actualiser les connaissances, pour le meilleur et pour le pire, peut-être. La philosophie spencérienne nous choque aujourd'hui par son manque de compassion. De tels scrupules ne venaient pas nécessairement à l'esprit des lecteurs informés de la fin du XIX ${ }^{e}$ siècle, comme des professeurs, inspecteurs, médecins, etc.

Que retient Georges Poyer, auteur de l'article « Spencer » dans le dictionnaire de 1911 ? Il se réfère naturellement à l'ouvrage intitulé De l'éducation intellectuelle, morale et physique; il dit à propos des idées de Spencer :

Elles font réfléchir, elles ont agi comme un stimulant, et c'est sans doute par là que s'explique le succès qu'a remporté en France particulièrement, ce petit livre si plein de verve, si savoureux parfois, et pourtant toujours pénétré de la gravité et de l'importance du sujet qu'il traite. ${ }^{17}$

Dans le détail, que retient-il ? Le premier des articles qui composent le recueil, «Quelle est la connaissance la plus utile?", est pour l'auteur l'apport majeur de Spencer à la pensée de l'éducation. Il en retient les éléments suivants : tout d'abord, la critique du système d'éducation traditionnel, qu'on peut définir pour aller vite comme l'héritage des humanités, que Spencer déclare périmé au nom du critère de l'utilité ${ }^{18}$. Ce qui peut favoriser la vie humaine et sa réalisation sous tous ses aspects, selon les propos de Spencer, doit être placé en premier. Les avancées des sciences de la nature donnent à 
l'homme un pouvoir inédit sur sa santé. L'hygiène doit donc prendre le relais de l'instinct de conservation, connaissance innée que, pour Spencer, on doit laisser s'épanouir chez l'enfant en respectant son besoin d'activité spontanée. Les connaissances scientifiques qui permettent d'étendre le pouvoir de l'humanité sur la nature sont éminemment souhaitables, alors que l'éducation esthétique et littéraire vient au dernier rang, comme quelque chose qui n'est pas indispensable au bonheur.

Georges Poyer voit dans cette doctrine la préfiguration de l'enseignement moderne récemment mis en place dans les lycées; il y adhère avec peu de réserves; il en déplore simplement les excès, en invoquant l'importance de l'« esprit de finesse ", de la notion d'" honnête homme", sans paraitre s'apercevoir que ces notions sont si antinomiques avec la conception spencérienne que leur réintroduction dans une pédagogie inspirée de celle-ci relève de l'incohérence. C'est qu'il prend à la lettre les propos de Spencer luimême, sans y voir l'auteur d'un système, dans lequel il faut replacer sa pensée de l'éducation si l'on veut véritablement la comprendre.

Quant aux méthodes pédagogiques préconisées dans De l'éducation, Georges Poyer montre qu'il n'a pas perçu les enjeux, pourtant fondamentaux, du progressisme affiché par Spencer, dans sa référence aux idées de Rousseau et de Pestalozzi. Georges Poyer se contente d'adhérer à ces idées, en précisant que ce n'est pas l'aspect le plus original de la pensée de Spencer $!^{19}$

20 À côté de cette adhésion que l'on pourrait qualifier de "molle», une opposition structurée à l'influence de Spencer dans le domaine de l'éducation se constitue dans les milieux néo-kantiens des disciples de Charles Renouvier. Leurs critiques se concentrent sur le problème de la morale. En effet, la montée en puissance du paradigme évolutionniste tend à renforcer les critiques matérialistes de la morale. Les idéaux, les valeurs, les croyances s'y trouvent ravalés ensemble au statut de productions provisoires d'un esprit humain par ailleurs variable, en évolution. Le fondement des valeurs s'en trouve relativisé, les règles de la conduite, comme on le voit très bien chez Spencer, se trouvent réduites à des adaptations d'inégale valeur qu'il convient de changer. Les nouveaux adeptes de Kant accordent au contraire une spécificité à l'esprit humain par rapport aux autres vivants, militent pour une indépendance de la morale par rapport à la science, et voient dans la reviviscence de la morale kantienne une alternative à l'empire hégémonique des sciences de la nature, non seulement dans la connaissance, mais encore dans le domaine de la métaphysique et dans celui de la pratique.

Une telle inspiration se retrouve à l'intérieur de l'institution scolaire, par exemple à travers la remarquable personnalité d'Octave Gréard (1828-1904). C'est un haut administrateur, responsable de la nouvelle organisation pédagogique de l'enseignement primaire. Il est normalien, agrégé de Lettres, directeur de l'enseignement primaire de la Seine en 1870, inspecteur général de l'Instruction publique en 1871, directeur au ministère de l'Instruction publique en 1872, vice-recteur de l'académie de Paris jusqu'en 1902... Partisan de la méthode intuitive de Pestalozzi, on ne peut le considérer comme un tenant de la tradition ou un partisan des méthodes autoritaristes, même s'il incarne incontestablement l'autorité par ses fonctions officielles.

Octave Gréard s'est illustré dans une discussion extrêmement précise sur la conception de la discipline de Spencer, des "réactions naturelles ». Se ressourçant auprès des auteurs les plus lointains, tels Quintilien, comme si la contestation de Spencer impliquait un ressaisissement de la philosophie de l'éducation depuis le départ, il montre que cette 
philosophie est aux antipodes des valeurs républicaines. Pour lui il y a antinomie entre le désir de voir réussir les meilleurs, au sens où les meilleurs sont ceux qui s'imposent l'effort et contribuent au bien commun, et la notion évolutionniste de "concurrence", qui mène à considérer l'autre comme un ennemi, à le vaincre par tous les moyens. C'est pourquoi, au-delà de l'évolutionnisme proprement dit, Octave Gréard vise l'idée de nature ${ }^{20}$, il critique conjointement Rousseau et Spencer, leur reprochant de prendre la nature pour guide. Pour lui, l'institution scolaire doit demeurer un moyen d'éduquer, au sens de diriger, le développement de l'enfant, en tenant compte de sa nature, certes, mais en visant également un idéal moral construit par la civilisation. Le fait de réunir les enfants à l'école, et plus particulièrement dans la classe, constitue une situation éducative par ellemême, mais à condition de bien distinguer l'«émulation" de la "concurrence ». L'émulation implique que chacun prenne modèle sur les meilleurs pour se dépasser soimême; cette situation ne comporte pas à proprement parler de lutte, encore moins nécessite-t-elle la sélection ou l'élimination. La concurrence, au contraire, chère aux évolutionnistes, implique qu'il y ait des premiers et des derniers, elle dresse forcément les individus les uns contre les autres. À l'aide de cette distinction, Octave Gréard oppose une pédagogie libertaire qui prend le risque, au nom de l'argument de la liberté, de mettre les individus en concurrence, à une pédagogie fondée sur l'autorité, qui malgré les apparences, promeut véritablement la liberté en augmentant le pouvoir de l'individu, à commencer par le pouvoir qu'il conquiert sur lui-même, le pouvoir de l'effort, le pouvoir d'apprendre. Gréard considère que la nature humaine a d'autres exigences concernant le développement de la liberté, que la spontanéité pure ne saurait y suffire. Si la liberté fait partie de l'essence de l'homme, elle n'est pas un produit de la nature et de l'évolution. C'est une vision différente de la société qui est en jeu à travers cette discussion. En effet, l'école de Gréard s'articule au projet d'une élite distinguée par son mérite, c'est-à-dire ses talents et sa moralité, qui serait l'aristocratie du monde moderne. En rupture avec les vieilles hiérarchies autoritaires, symboliques, arbitraires, la démocratie moderne articulerait la reconnaissance des qualités individuelles et l'intérêt général. Il faut remarquer que, dans sa critique, Gréard admet la filiation revendiquée par Spencer, et il interprète l'éloge fait par ce dernier de la concurrence vitale comme une suite logique de l'individualisme de Rousseau ${ }^{21}$. Il impute donc indistinctement aux deux philosophes la responsabilité des idées qu'il réprouve: si l'individu ne se compare qu'à lui-même et n'obéit à personne, c'est pour se trouver livré à la brutalité de la lutte pour l'existence, où la seule légitimité de la réussite est la réussite elle-même. Ceci mène selon Gréard au chaos, à une juxtaposition d'individus qui ne font pas une société. Cependant, cela ne l'empêche pas de reconnaitre comme un fait l'aspiration générale à la liberté individuelle, et le fait que les rapports humains sont de moins en moins régis par l'autorité.

On peut se demander pourquoi, en dépit d'une discussion si approfondie, la philosophie de Spencer a continué, non seulement à avoir du succès, mais encore à exercer une espèce de fascination? Pourquoi, en dépit de ses conséquences potentiellement dangereuses et de son argumentation scientifique contestable, a-t-elle pu incarner à ce point la Vérité ? Les convictions sont plus puissantes que les théories qui les incarnent, plus puissantes aussi que les réfutations de ces idées. Derrière les convictions, des affects et des valeurs jouent de manière implicite. Le succès des idées spencériennes, parfois comme on a pu le voir jusqu'à nos jours, tiennent à la victoire progressive d'une telle conviction : celle que l'individu doit tout devoir à lui-même, et qu'il ne doit plus être redevable aux générations antérieures. Cette certitude est scellée par une autre: demain sera différent d'aujourd'hui, alors qu'aujourd'hui ressemblait encore à hier. Ce mouvement de refus de 
toute tradition et de toute dette, de la transmission par la relation éducative, se heurte à présent à la désillusion et à l'inquiétude concernant l'avenir du progrès. Il serait donc important de reprendre à nouveaux frais les discussions inachevées ou abandonnées autour des idées spencériennes sur l'éducation, qui avaient le mérite de théoriser ce qui nous apparait aujourd'hui comme indiscutable, même si ses convictions usurpaient parfois l'autorité de la science.

\section{NOTES}

1. Herbert Spencer, Education intellectual, moral and physical, Londres, Manwarning, 1861.

2. Félix Pécaut, « De l'usage et de l'abus de la pédagogie », Revue pédagogique, n 1, 1882, p. 97-110, p. 97.

3. Herbert Spencer, Les premiers principes, trad. Par E. Cazelles, Paris, G. Baillière, 1871 [1862].

4. On peut se référer pour une étude plus générale des concepts spencériens à Penser Spencer, Daniel Becquemont et Dominique Ottavi (dir.), Saint-Denis, Presses Universitaires de Vincennes, 2011.

5. Voir par exemple Michel Soëtard, M. Pestalozzi ou la naissance de l'éducateur. Berne, P. Lang, 1981.

6. Herbert Spencer, De l'éducation intellectuelle, morale et physique, chap. " De l'éducation morale ", Paris, Alcan, 6 édition, 1885 [1861], p. 198 sq.

7. Ibid., chap. « De l'éducation intellectuelle », p. 128 sq.

8. Ibid., chap. « De l'éducation physique », p. 233 sq.

9. Ibid., chap. « De l'éducation morale », p. 198 sq.

10. Ibid., p. 153 : «[...] nous avons entendu parler d'une école de filles dans laquelle plusieurs élèves s'occupent volontairement de géométrie en dehors des heures de classe [...]».

11. Herbert Spencer, Introduction à la science sociale, Paris, Germer Baillière, 1874, chap. «Les préjugés de l'éducation », p. 59-79.

12. Théodule Ribot a consacré un chapitre à Spencer dans son ouvrage sur La Psychologie anglaise contemporaine, Paris, Librairie philosophique Ladrange, 1870.

13. Dominique Ottavi, De Darwin à Piaget : pour une histoire de la psychologie de l'enfant, Paris, CNRS éditions, $2^{\mathrm{e}}$ éd., 2009 [2001].

14. Paul Souquet, «De l'éducation intellectuelle, morale et physique », Revue pédagogique, $\mathrm{n}^{\circ} 2$, 1880, p. 328-350.

15. Henri Gazin, «Le système des réactions naturelles d'après Herbert Spencer ", Revue pédagogique, $\mathrm{n}^{\circ} 1,1911$, p. 27-40.

16. Voir par exemple Eirick Prairat, Éduquer et punir, Nancy, Presses Universitaires de Nancy, 1994.

17. Cf. l'entrée «Spencer (Herbert)» dans l'édition de 1911 du Dictionnaire de pédagogie de Ferdinand Buisson, qui est accessible en ligne sur le site de l'Institut Français d'Éducation: http://www.inrp.fr/edition-electronique/lodel/dictionnaire-ferdinand-buisson/document.php? id=3657 (page consultée le 7 mai 2014).

18. Herbert Spencer, Education intellectual, moral and physical, op.cit., p. 1-88. 
19. «Les idées de Spencer sur la façon d'organiser l'éducation intellectuelle sont beaucoup moins originales. Il suit ici presque constamment Rousseau et Pestalozzi.»; entrée «Spencer (Herbert) », op. cit.

20. Voir notamment Octave Gréard, «L'esprit de discipline dans l'éducation », Revue pédagogique, $\mathrm{n}^{\circ} 1,1883$, p. 385-446.

21. Ibid., p. 395 : « C'est le pur système de Rousseau... ».

INDEX

Mots-clés : éducation, pédagogie, lutte pour l'existence

\section{AUTEUR}

DOMINIQUE OTTAVI

Paris-Ouest Nanterre 